\title{
Ranking scientists and departments in a consistent manner
}

\author{
Denis Bouyssou* Thierry Marchant ${ }^{\dagger}$
}

January 25, 2011

\begin{abstract}
The standard data that we use when computing bibliometric rankings of scientists are just their publication/citation records, i.e., so many papers with 0 citation, so many with 1 citation, so many with 2 citations, etc. The standard data for bibliometric rankings of departments have the same structure. It is therefore tempting (and many authors gave in to temptation) to use the same method for computing rankings of scientists and rankings of departments. Depending on the method, this can yield quite surprising and unpleasant results. Indeed, with some methods, it may happen that the "best" department contains the "worst" scientists, and only them. This problem will not occur if the rankings satisfy a property called consistency, recently introduced in the literature. In this paper, we explore the consequences of consistency and we characterize two families of consistent rankings.
\end{abstract}

\section{Introduction}

Several recent papers [Waltman and van Eck, 2009, Waltman et al., 2011, Marchant, 2009a,b, Bouyssou and Marchant, 2011] have independently discussed the importance of a property called Independence for bibliometric rankings of scientists. This property (called consistency by Waltman and van Eck [2009]) states that, whenever two scientists improve their publication/citation records by the same amount, then they should compare in the same manner after and before the improvement. Consider for instance two scientists $f$ and $g$ and suppose that, in a

Authors are listed alphabetically. They have contributed equally to the text.

*CNRS and Université Paris Dauphine. Email: bouyssou@lamsade.dauphine.fr

†Ghent University. Email: thierry.marchant@ugent.be 
given ranking, $f$ is better than $g$. Suppose that $f$ and $g$ both publish two extra papers, each one being cited five times. After this improvement, they are denoted by $f^{\prime}$ and $g^{\prime}$. Then a ranking satisfying Independence will rank $f^{\prime}$ and $g^{\prime}$ in the same way as $f$ and $g$, that is, $f^{\prime}$ better than $g^{\prime}$. It is well known [Marchant, 2009b] that the ranking of authors based on the h-index violates independence. ${ }^{1}$ This is illustrated below.

Example 1 Consider two authors. The first one has four papers with four citations each. The second has three papers with six citation each. The h-index of the first author is 4, while the h-index of second is 3. Hence the first author is judged better than the second one.

Suppose that each of the above authors publish an additional paper with six citations. The h-index of the two authors is now 4, so that they are now judged equivalent.

Finally, if both authors now publish an additional paper with six citations, the $h$-index of the first author stays at 4, while the h-index of the second raises at 5. The second author is now judged better than the first one.

When we simultaneously consider rankings of scientists and rankings of departments (or universities, schools, ... ), Waltman and van Eck [2009] have shown that Independence is related to another property that we call Consistency: if two departments have the same size (say 2), if the first scientist in department 1 is better that the first scientist in department 2 and if the second scientist in department 1 is better that the second scientist in department 2 , then department 1 should be better than department 2. As shown below, this property is violated if scientists and departments are ranked according to their h-index.

Example 2 Consider two departments each consisting of two scientists. The scientists in department 1 both have 4 papers, each one cited 4 times. The scientists in department 2 both have 3 papers, each one cited 6 times.

Both scientists in department 1 have an h-index of 4 and are therefore better than both scientists in department 2, with an h-index of 3. Yet, department 1 has an h-index of 4 and is therefore worse than department 2 with an h-index of 6. Hence, the "best" department contains the "worst" scientists.

Because of the somewhat informal character of their paper, Waltman and van Eck [2009] do not make a clear distinction between Independence and Consistency (they use the same name for both). They show that these two properties are intuitively very close to each other but they do not clearly establish the logical relations between them. The purpose of this paper is to pursue Waltman and van Eck's

\footnotetext{
${ }^{1}$ The same is true for rankings that are based on an "average score", e.g., the average number of citations per paper published.
} 
idea and to formally analyze the consequences (for scientists and departments) of imposing Consistency.

After some notation and definitions (Section 2), we analyze the relation bewteen Independence and Consistency in Section 3. We show that Consistency does not imply Independence unless an additional condition (Totality) is imposed. In Section 4, we explore the consequences of imposing Consistency and Totality as well as some other conditions. This leads us to characterize in Section 4 a family of rankings of scientists (the so-called scoring rules, already analyzed in Marchant [2009b]) and two families of rankings of departments. The first one is close to scoring rules for scientists: it is based on a kind of total score. The second one is slightly different in that it is based on a kind of average score. Section 5 proves the independence of the conditions used in the characterizations, Section 6 factorizes Totality in several more elementary axioms and Section 7 concludes.

\section{Notation and definitions}

We represent a scientist by a mapping $f$ from $\mathbb{N}=\{0,1,2, \ldots\}$ to $\mathbb{N}$ and we interpret $f(x)$ as the number of publications of scientist $f$ with exactly $x$ citations. $^{2}$ The number of publications of scientist $f$ is therefore $\sum_{x \in \mathbb{N}} f(x)$. We can also compute the total number of citations of scientist $f$ : it is given by $\sum_{x \in \mathbb{N}} x f(x)$. So, we actually do not represent a scientist as a person but we identify a scientist with his/her publication/citation records as modelled by $f$. Consider for instance a scientist that has published 5 papers. One of them is not cited, two are cited once, and the last two are cited 4 times. This scientist is viewed in our model as the function $f$ such that $f(0)=1, f(1)=2, f(4)=2$ and $f(x)=0$ for every $x \notin\{0,1,4\}$.

Let $X$ be the set all mappings $f$ from $\mathbb{N}$ to $\mathbb{N}$ such that

$$
\sum_{x \in \mathbb{N}} f(x) \text { is finite. }
$$

The set $X$ is called the set of scientists. The elements of $X$ are usually denoted by $f, f^{\prime}, g, f_{1}, \ldots$ Restriction (1) is very sensible since it guarantees that we will never face a scientist with infinitely many publications.

In this paper, we will investigate how we can construct a ranking (a complete and transitive binary relation ${ }^{3}$ ) $\succsim$ on $X$. The statement ' $f \succsim g$ ' is interpreted as 'given their publication/citation records, scientist $f$ is at least as good as scientist

\footnotetext{
${ }^{2}$ This formalism does not permit to handle coauthorship and multiple affiliation. This problem will be touched upon in the discussion.

${ }^{3} \mathrm{~A}$ binary relation $\succsim$ on the set $X$ is transitive if, $\forall f, g, h \in X, f \succsim g$ and $g \succsim h$ imply $f \succsim h$. It is complete if, $\forall f, g \in X, f \succsim g$ or $g \succsim f$.
} 
$g$.' When $f \succsim g$ and $g \succsim f$, we write $f \succ g$ ( $f$ is strictly better than $g$ ). When $f \succsim g$ and $g \succsim f$, we write $f \sim g$ ( $f$ and $g$ are equivalent $)$. The relations $\succ$ and $\sim$ are respectively named the asymmetric part and the symmetric part of $\succsim$.

A department will be represented by a vector of scientists ${ }^{4}$. The set of all departments of size $k$ is denoted $Y_{k}$. It is formally defined by $Y_{k}=\left\{\left(f_{1}, \ldots, f_{k}\right) \in\right.$ $\left.X^{k}\right\}$. The set of all departments is $Y=\bigcup_{k \in \mathbb{N}^{+}} Y_{k}$, where $\mathbb{N}^{+}$denotes the set of positive integers. In this paper, we are not only interested in a ranking $\succsim$ on $X$, but also in a ranking $\unrhd$ on $Y$, with symmetric and asymmetric parts respectively denoted by $\triangleq$ and $\triangleright$.

For all $x \in \mathbb{N}$, we denote by $\mathbf{1}_{x}$ the scientist such that $\mathbf{1}_{x}\left(x^{\prime}\right)=0$ whenever $x^{\prime} \neq x$ and $\mathbf{1}_{x}(x)=1$. So, $\mathbf{1}_{x}$ represents a scientist with exactly 1 publication, cited $x$ times. A scientist without publication is represented by $\mathbf{0}$.

\section{Consistency and Independence}

Rankings of scientists and rankings of departments should not be unrelated. It is common sense that a good department should consist of good scientists. The following axiom makes this explicit.

A 1 Consistency. If $f_{i} \succsim g_{i}$ for all $i \in\{1, \ldots, k\}$, then $\left(f_{1}, \ldots, f_{k}\right) \unrhd\left(g_{1}, \ldots, g_{k}\right)$. If, in addition, $f_{i} \succ g_{i}$ for some $i \in\{1, \ldots, k\}$, then $\left(f_{1}, \ldots, f_{k}\right) \triangleright\left(g_{1}, \ldots, g_{k}\right)$.

Notice that the statement of this condition involves the ranking $\succsim$ and the ranking $\unrhd$. It links both rankings in the following way: if the $i$-th scientist in department $\left(f_{1}, \ldots, f_{k}\right)$ is better than the $i$-th scientist in department $\left(g_{1}, \ldots, g_{k}\right)$ and this holds for all $i$ in $\{1, \ldots, k\}$, then department $\left(f_{1}, \ldots, f_{k}\right)$ must be better than department $\left(g_{1}, \ldots, g_{k}\right)$. Notice that both departments in this condition have the same size. We now present an axiom involving only $\succsim$.

A 2 Independence. For all $f, g \in X$, all $x \in \mathbb{N}, f \succsim g$ iff $f+\mathbf{1}_{x} \succsim g+\mathbf{1}_{x}$.

In the statement of this axiom, $f+\mathbf{1}_{x}$ is the sum of two functions. It is therefore a function and also represents a scientist. Intuitively, Independence can be understood as follows. Suppose a scientist $f$ is at least as good as $g$. Suppose also both of them publish one additional paper with the same number of citations. So, both make the same improvement. Then these two scientists (now represented by $f+\mathbf{1}_{x}$ and $g+\mathbf{1}_{x}$ ) should compare in the same way as previously, i.e., $f+\mathbf{1}_{x}$ is at least as good as $g+\mathbf{1}_{x}$. An apparently stronger condition than Independence is

\footnotetext{
${ }^{4} \mathrm{~A}$ department cannot be represented by a set of scientists because it would then be impossible to have a department containing two scientists with the same publication/citation records. That is why we use a vector. A multiset would also be possible.
} 
A 3 Strong Independence. For all $f, g, h \in X, f \succsim g$ iff $f+h \succsim g+h$.

This condition is called Consistency by Waltman and van Eck [2009]. It is obvious that Strong Independence implies Independence, but, actually, in our setting, Independence also implies Strong Independence, so that both conditions are logically equivalent.

Lemma 1 Let $\succsim$ be a ranking of scientists defined on $X$. It satisfies Independence if and only if it satisfies Strong Independence.

Proof. We only prove that Independence implies Strong Independence. Because of the definition of $X$, any scientist $h$ can be written as $h=\sum_{x \in \mathbb{N}} h(x) \mathbf{1}_{x}$. Let us start with $f \succsim g$. If we apply $h(0)$ times Independence, we obtain

$$
f+h(0) \mathbf{1}_{0} \succsim g+h(0) \mathbf{1}_{0} .
$$

By Independence again,

$$
f+h(0) \mathbf{1}_{0}+h(1) \mathbf{1}_{1} \succsim g+h(0) \mathbf{1}_{0}+h(1) \mathbf{1}_{1} .
$$

If we repeat this operation for every value of $x \in \mathbb{N}$ such that $h(x) \neq 0$, we find

$$
f+\sum_{x \in \mathbb{N}} h(x) \mathbf{1}_{x} \succsim g+\sum_{x \in \mathbb{N}} h(x) \mathbf{1}_{x}
$$

i.e., $f+h \succsim g+h$.

Because of this lemma, we will speak only of Independence in the sequel. Waltman and van Eck [2009] have noticed that Independence and Consistency are closely related and they have even suggested that a ranking violating Independence would also violate Consistency. They provide an example supporting this: the ranking of scientists based on the h-index violates Independence (see also Example 1 above) and the ranking of departments based on the h-index violates Consistency (see also Example 2 above). Actually, since $\succsim$ and $\unrhd$ are two distinct rankings (this is not explicit in Waltman and van Eck's paper), a violation of Independence (a property involving only $\succsim$ and scientists) has no direct impact on the properties of $\unrhd$. There are indeed pairs of rankings $(\succsim, \unrhd)$ such that Consistency holds but not Independence: for instance, the ranking of scientists $\succsim$ based on the h-index and the ranking of departments $\unrhd$ based on the sum over its members of their h-index.

Nevertheless Waltman and van Eck's intuition is correct, even though it rides on a hidden assumption that we now make explicit. 
A 4 Totality. Let $k \in \mathbb{N}^{+}$and $\left(f_{1}, f_{2}, \ldots, f_{k}\right),\left(g_{1}, g_{2}, \ldots, g_{k}\right)$ be two departments in $Y_{k}$. If $\sum_{i=1}^{k} f_{i}=\sum_{i=1}^{k} g_{i}$, then $\left(f_{1}, \ldots, f_{k}\right) \triangleq\left(g_{1}, \ldots, g_{k}\right)$.

Notice that $\sum_{i=1}^{k} f_{i}$ is the aggregated publication/citation record of the whole department $\left(f_{1}, \ldots, f_{k}\right)$. So, Totality says that, whenever two equal sized departments have the same citation distribution, then they must be equivalent. In other words, in order to evaluate a department, we do not need to know who published what and was cited $x$ times, we just need to know the total publication/citation record of the department. Unlike Consistency, that we find very compelling, Totality might be subject to discussion. We could indeed consider that two departments with the same total publication/citation record but with a different distribution among members should not necessarily be ranked as indifferent. We could for example favour the department with the most uniform distribution. Notice that, although we have no statistics on this, it seems that most people using rankings of departments abide by Totality : they compute their ranking using only $\sum_{i=1}^{k} f_{i}$ and not the distribution among members [e.g., Coupé, 2003, Dusansky and Vernon, 1998, Kinney, 2007, Kinnucan and Traxler, 1994]. We found only two exceptions [Schubert, 2007, Arencibia-Jorge et al., 2008] : for each department, they compute a h-index which is not based on the total publication/citation record but on the distribution of the h-indices of the scientists within the department. We are now ready to formally state and prove Waltman and van Eck's intuition.

Proposition 1 If $\succsim$ and $\unrhd$ are linked by Consistency and if $\unrhd$ satisfies Totality, then $\succsim$ satisfies Independence.

Proof. By Totality, for all $f, g, h \in X$, we have $(f, g+h) \triangleq(g, f+h)$. We must prove $f \succsim g \Longleftrightarrow f+h \succsim g+h$. Proof of $\Rightarrow$. Suppose $f \succsim g$. Suppose also, contrary to Independence, $g+h \succ f+h$. Then Consistency implies $(f, g+h) \triangleright(g, f+h)$, violating Totality. Proof of $\Leftarrow$. Suppose now $f+h \succsim g+h$ and, contrary to Independence, $g \succ f$. Then Consistency imply $(f+h, g) \triangleright(g+h, f)$, violating again Totality.

If we apply this proposition to the ranking $\succsim$ based on the h-index, following Waltman and van Eck, we obtain

Corollary 1 If $\succsim$ is the ranking of scientists based on the h-index, then there is no ranking $\unrhd$ such that Totality and Consistency hold.

Proof. By Proposition 1, Totality and Consistency imply Independence but Example 1 has shown that the ranking based on the h-index does not satisfy Independence. 
The h-index can be replaced in the statement of Corollary 1 by the g-index [Egghe, 2006a,b], the h(2)-index [Kosmulski, 2006], the R-index [Jin et al., 2007], the $\mathrm{h}_{T}$-index [Anderson et al., 2008], and the $\mathrm{h}_{\alpha^{-}}$and $\mathrm{g}_{\alpha}$-indices [van Eck and Waltman, 2008], since Waltman and van Eck [2009] have noticed that none of the rankings based on these indices satisfies Independence.

We are convinced that Consistency is a very appealing condition and that Totality may make sense in many circumstances. In the next sections, we will therefore further analyze the consequences of imposing these two conditions.

\section{Scoring rules and averaging rules}

We say that a ranking $\succsim$ is a scoring rule for scientists (s-scoring rule for short) if there is a mapping $u: \mathbb{N} \rightarrow \mathbb{R}: x \rightarrow u(x)$ such that

$$
f \succsim g \Longleftrightarrow \sum_{x \in \mathbb{N}} f(x) u(x) \geq \sum_{x \in \mathbb{N}} g(x) u(x) .
$$

In this expression, $u(x)$ represents the value or the score of one publication with $x$ citations. We multiply $u(x)$ by the number of papers with $x$ citations and we obtain the product $f(x) u(x)$. The sum then represents the total score of a scientist. Many popular bibliometric rankings are s-scoring rules and correspond to an appropriate choice of the mapping $u$ [see Marchant, 2009b]. We present just one example: if $u(x)=x$, then $\sum_{x \in \mathbb{N}} f(x) u(x)$ is the total number of citations of scientist $f$ and the ranking $\succsim$ is in decreasing order of total number of citations. Some rankings of scientists do not belong to the family of s-scoring rules: for instance, the ranking based on the h-index [Hirsch, 2005] or the ranking based on the average number of citations per paper [van Raan, 2006]. It is easy to check that all scoring rules satisfy Independence.

We say that a ranking $\unrhd$ is a scoring rule for departments (d-scoring rule for short) if there is a mapping $v: \mathbb{N} \rightarrow \mathbb{R}: x \rightarrow v(x)$ such that

$$
\left(f_{1}, \ldots, f_{k}\right) \unrhd\left(g_{1}, \ldots, g_{\ell}\right) \Longleftrightarrow \sum_{i=1}^{k} \sum_{x \in \mathbb{N}} f_{i}(x) v(x) \geq \sum_{i=1}^{\ell} \sum_{x \in \mathbb{N}} g_{i}(x) v(x) .
$$

Here is an example of a d-scoring rule: if $v(x)=x$, then $\sum_{i=1}^{k} \sum_{x \in \mathbb{N}} f_{i}(x) v(x)$ is the total number of citations of department $\left(f_{1}, \ldots, f_{k}\right)$ and the ranking $\unrhd$ is in decreasing order of total number of citations.

We say that a ranking of departments $\unrhd$ is an averaging rule for departments (d-averaging rule for short) if there is a mapping $v: \mathbb{N} \rightarrow \mathbb{R}: x \rightarrow v(x)$ such that

$$
\left(f_{1}, \ldots, f_{k}\right) \unrhd\left(g_{1}, \ldots, g_{\ell}\right) \Longleftrightarrow \frac{1}{k} \sum_{i=1}^{k} \sum_{x \in \mathbb{N}} f_{i}(x) v(x) \geq \frac{1}{\ell} \sum_{i=1}^{\ell} \sum_{x \in \mathbb{N}} g_{i}(x) v(x) .
$$


An appropriate choice of the mapping $v$ yields different rankings that are used in practice. For instance, if $v(x)=x$, then $\frac{1}{k} \sum_{i=1}^{k} \sum_{x \in \mathbb{N}} f_{i}(x) v(x)$ is the average (per scientist) number of citations of department $\left(f_{1}, \ldots, f_{k}\right)$ and the ranking $\unrhd$ is in decreasing order of average number of citations.

Here is now a simple lemma about the families we just defined.

Lemma 2 If $\succsim$ is a s-scoring rule and $\unrhd$ is a $d$-scoring rule or a d-averaging rule with $u=v$, then they are linked by Consistency and $\unrhd$ satisfies Totality.

Proof. Let us first prove that Totality holds. Suppose that $\sum_{i=1}^{k} f_{i}=\sum_{i=1}^{k} g_{i}$, i.e., for all $x \in \mathbb{N}, \sum_{i=1}^{k} f_{i}(x)=\sum_{i=1}^{k} g_{i}(x)$. Then

$$
\begin{aligned}
\sum_{i=1}^{k} \sum_{x \in \mathbb{N}} f_{i}(x) v(x) & =\sum_{x \in \mathbb{N}} v(x) \sum_{i=1}^{k} f_{i}(x) \\
& =\sum_{x \in \mathbb{N}} v(x) \sum_{i=1}^{k} g_{i}(x) \\
& =\sum_{i=1}^{k} \sum_{x \in \mathbb{N}} g_{i}(x) v(x)
\end{aligned}
$$

and, hence, $\left(f_{1}, \ldots, f_{k}\right) \triangleq\left(g_{1}, \ldots, g_{k}\right)$. A similar reasoning applies for d-averaging rules.

We now prove that Consistency holds. Suppose $f_{i} \succsim g_{i}$ for all $i \in\{1, \ldots, k\}$, $\succsim$ is a s-scoring rule and $\unrhd$ is a d-scoring rule with $u=v$. From $f_{i} \succsim g_{i}$, we obtain

$$
\begin{aligned}
\sum_{x \in \mathbb{N}} f_{1}(x) v(x) & \geq \sum_{x \in \mathbb{N}} g_{1}(x) v(x), \\
& \vdots \\
\sum_{x \in \mathbb{N}} f_{i}(x) v(x) & \geq \sum_{x \in \mathbb{N}} g_{i}(x) v(x), \\
& \vdots \\
\sum_{x \in \mathbb{N}} f_{k}(x) v(x) & \geq \sum_{x \in \mathbb{N}} g_{k}(x) v(x) .
\end{aligned}
$$

Adding these inequalities, we find

$$
\sum_{i=1}^{k} \sum_{x \in \mathbb{N}} f_{i}(x) v(x) \geq \sum_{i=1}^{k} \sum_{x \in \mathbb{N}} g_{i}(x) v(x),
$$


Then, by definition of a d-scoring rule, $\left(f_{1}, \ldots, f_{k}\right) \unrhd\left(g_{1}, \ldots, g_{k}\right)$. Furthermore, if any of the above inequalities is strict, we obtain

$$
\sum_{i=1}^{k} \sum_{x \in \mathbb{N}} f_{i}(x) v(x)>\sum_{i=1}^{k} \sum_{x \in \mathbb{N}} g_{i}(x) v(x),
$$

so that, using the definition of a d-scoring rule, $\left(f_{1}, f_{2}, \ldots, f_{k}\right) \triangleright\left(g_{1}, g_{2}, \ldots, g_{k}\right)$. This proves that Consistency holds. A similar reasoning applies for d-averaging rules.

The distinction between d-scoring rules and d-averaging rules may remind you the distinction between total performance index and average performance index in Waltman et al. [2011], Waltman and van Eck [2009]. But an average performance index is an average per paper while a d-averaging rule is based on an average per scientist.

\section{1 s-scoring rules and d-scoring rules}

We now turn to the characterization of the pairs of rankings $(\succsim, \unrhd)$ where $\succsim$ is a s-scoring rule and $\unrhd$ is a d-scoring rule with $u=v$. We will need two more conditions.

A 5 Archimedeanness. For all $f, g, h, e \in X$ with $f \succ g$, there is an integer $n$ such that $e+n \cdot f \succsim h+n \cdot g$.

In this condition, $n \cdot g$ is standard product of a function by a number. It is clear that this new function $n \cdot g$ belongs to $X$ and therefore represents a scientist. Let us try to explain the intuitive content of this condition. Suppose $f \succ g$ and $e \prec h$. Let us add $f$ and $e$, on the one hand, and $g$ and $h$ on the other hand. It can happen that the difference between $f$ and $g$ is so large that it compensates the difference between $e$ and $h$. In that case, we have $f+e \succsim g+h$. Suppose now this is not the case. Then repeating the same operation, we might have $f+f+e \succsim g+g+h$. Suppose this is still not the case. Then perhaps $f+f+f+e \succsim g+g+g+h$. The Archimedean condition says that keeping adding $f$ and $g$ will necessarily lead to $f+\ldots+f+e \succsim g+\ldots+g+h$ because the difference between $f+\ldots+f$ and $g+\ldots+g$ gets larger and larger.

All scoring rules for scientists clearly satisfy Archimedeanness. The interested reader will find some examples of rankings violating Archimedeannness in Marchant [2009b]. The h-index is one of them. Our next condition is about the impact of scientists without publications on the ranking of departments.

A 6 Dummy Scientist. For all $f_{1}, \ldots, f_{k} \in X,\left(f_{1}, \ldots, f_{k}\right) \triangleq\left(f_{1}, \ldots, f_{k}, \mathbf{0}\right)$. 
In other words, adding a dummy scientist (without any paper) to a department does not improve nor worsen its position. Notice that this condition is exclusively about $\unrhd$. It is satisfied by d-scoring rules but not by d-averaging rules. We are now ready to present a characterization of pairs of rankings $(\succsim, \unrhd)$, where $\succsim$ is a scoring rule and $\unrhd$ is a scoring rule.

Theorem 1 Let $\succsim$ and $\unrhd$ be respectively a ranking of all scientists in $X$ and a ranking of all departments in $Y$. The relations $\succsim$ and $\unrhd$ are linked by Consistency (A1), $\unrhd$ satisfies Totality (A4) and Dummy Scientist (A6), and $\succsim$ satisfies Archimedeanness (A5) if and only $\succsim$ is a s-scoring rule and $\unrhd$ is a d-scoring rule, with $u=v$. Futhermore, the mapping $u$ is unique up to a positive linear transformation. ${ }^{5}$

Before proving this theorem, we recall a standard theorem in Measurement Theory [Luce, 2000, Th. 4.3.2, p.144].

Theorem 2 Let $R$ be a binary relation on a set $A$. The asymmetric (resp. symmetric) part of $R$ is denoted by $P$ (resp. I). Let $\circ$ be a closed binary operation on A. For all $a \in A$ and $n>1$, define $a(1)=a$ and $a(n)=a(n-1) \circ a$. The triple $(A, R, \circ)$ satisfies

(i) $R$ is transitive and complete;

(ii) $\forall a, b, c \in A, a R b$ iff $a \circ c R b \circ c$;

(iii) $\forall a, b, c \in A, a \circ(b \circ c)=(a \circ b) \circ c$;

(iv) $\forall a, b, c, d \in A$ with a $P b$, there is an integer $n$ such that $a(n) \circ c R b(n) \circ d$;

(v) there is $\varepsilon \in A$ such that, for all $a \in A, \varepsilon \circ$ a $I$ a;

if and only if there is a mapping $\phi: A \rightarrow \mathbb{R}$ such that, for all $a, b \in A$,

- $a R b$ iff $\phi(a) \geq \phi(b)$

- $\phi(\varepsilon)=0$ and

- $\phi(a \circ b)=\phi(a)+\phi(b)$.

Furthermore, the mapping $\phi$ is unique up to a multiplication by a positive constant.

\footnotetext{
${ }^{5}$ In Marchant [2009b], we presented a somewhat similar theorem (only for $\succsim$ ). In that paper, the uniqueness is erroneously said to be up to a positive affine transformation. It should be up to a positive linear transformation.
} 
Proof of Theorem 1. It is easy to check that all conditions are necessary. We only prove the sufficiency. By Proposition $1, \succsim$ satisfies Independence. Let us now prove that $\succsim$ is a scoring rule ${ }^{6}$. To this end, we show that the triple $(X, \succsim,+)$ satisfies all conditions of Theorem 2, where the set $A$ is replaced by $X$, the relation $R$ by $\succsim$ and the binary operation $\circ$ by + . Condition $(i)$ is clearly satisfied because $\succsim$ is transitive and complete. Condition (ii) holds because of Independence. Indeed, suppose $f \succsim g$ and suppose $e$ is a scientist with $k$ publications. If we apply $k$ times Independence, we find $f+e \succsim g+e$. Condition (iii) is satisfied because the binary operation + on $X$ is associative. Condition ( $i v)$ holds because of Archimedeanness. Finally, it is easy to see that $\mathbf{0}$ is an identity for the operation + , just like $\varepsilon$ is an identity for $\circ$, so that condition $(v)$ is verified.

So, there is $\phi: X \rightarrow \mathbb{R}$ such that

$$
\begin{gathered}
f \succsim g \Longleftrightarrow \phi(f) \geq \phi(g), \\
\phi(\mathbf{0})=0
\end{gathered}
$$

and

$$
\phi(f+g)=\phi(f)+\phi(g) .
$$

Since any scientist $f \in X$ can be written as $\sum_{x \in \mathbb{N}} f(x) \mathbf{1}_{x}$, using (4), we find

$$
\phi(f)=\sum_{x \in \mathbb{N}} f(x) \phi\left(\mathbf{1}_{x}\right)
$$

If we now define $u(x)=\phi\left(\mathbf{1}_{x}\right)$, we can rewrite (2) as

$$
f \succsim g \Longleftrightarrow \sum_{x \in \mathbb{N}} f(x) u(x) \geq \sum_{x \in \mathbb{N}} g(x) u(x) .
$$

This completes the proof that $\succsim$ is a s-scoring rule.

Consider now two departments $\left(f_{1}, \ldots, f_{k}\right)$ and $\left(g_{1}, \ldots, g_{\ell}\right)$. Define $f=\sum_{i=1}^{k} f_{i}$ and $g=\sum_{i=1}^{\ell} g_{i}$. By Totality,

$$
(f, \underbrace{\mathbf{0}, \ldots, \mathbf{0}}_{k-1}) \triangleq\left(f_{1}, \ldots, f_{k}\right) \text { and }(g, \underbrace{\mathbf{0}, \ldots, \mathbf{0}}_{\ell-1}) \triangleq\left(g_{1}, \ldots, g_{\ell}\right) \text {. }
$$

Assume without loss of generality $\ell \geq k$. Using Dummy Scientist, we obtain

$$
(f, \underbrace{\mathbf{0}, \ldots, \mathbf{0}}_{\ell-1}) \triangleq(f, \underbrace{\mathbf{0}, \ldots, \mathbf{0}}_{k-1})
$$

\footnotetext{
${ }^{6}$ This part of the proof is very similar to the proof of Theorem 1 in Marchant [2009b]. There are two differences: (i) we do not take journals into account; (ii) the problem of coauthorship is not dealt with.
} 
and, by transitivity, we find

$$
(f, \underbrace{\mathbf{0}, \ldots, \mathbf{0}}_{\ell-1}) \triangleq\left(f_{1}, \ldots, f_{k}\right) .
$$

By transitivity,

$$
\left(f_{1}, \ldots, f_{k}\right) \unrhd\left(g_{1}, \ldots, g_{\ell}\right) \Longleftrightarrow(f, \underbrace{\mathbf{0}, \ldots, \mathbf{0}}_{\ell-1}) \unrhd(g, \underbrace{\mathbf{0}, \ldots, \mathbf{0}}_{\ell-1}) .
$$

Finally, by Consistency, $\left(f_{1}, \ldots, f_{k}\right) \unrhd\left(g_{1}, \ldots, g_{\ell}\right)$ iff $f \succsim g$ iff $\sum_{i=1}^{k} \sum_{x \in \mathbb{N}} f_{i}(x) u(x) \geq$ $\sum_{i=1}^{\ell} \sum_{x \in \mathbb{N}} g_{i}(x) u(x)$. This proves that $\unrhd$ is a d-scoring rule, with the same value function as $\succsim$.

\section{$4.2 \quad$ s-scoring rules and d-averaging rules}

We now turn to the characterization of the pairs of rankings $(\succsim, \unrhd)$ where $\succsim$ is a s-scoring rule and $\unrhd$ is a d-averaging rule with $u=v$. For that purpose, we need a new axiom.

A 7 Homogeneity. For all $f_{1}, \ldots, f_{k} \in X$ and all $n \in \mathbb{N} \backslash\{0\},\left(f_{1}, \ldots, f_{k}\right) \triangleq$ $(\underbrace{f_{1}, \ldots, f_{1}}_{n}, \ldots, \underbrace{f_{i}, \ldots, f_{i}}_{n}, \ldots, \underbrace{f_{k}, \ldots, f_{k}}_{n})$.

This amounts to saying that size (in terms of number of scientists) does not matter. Notice that this condition is exclusively about $\unrhd$. It is satisfied by d-averaging rules but not by d-scoring rules.

Theorem 3 Let $\succsim$ and $\unrhd$ be respectively a ranking of scientists and a ranking of departments. The relations $\succsim$ and $\unrhd$ are linked by Consistency (A1), $\unrhd$ satisfies Totality(A4) and Homogeneity (A7), and $\succsim$ satisfies Archimedeanness (A5) if and only if $\succsim$ is a s-scoring rule and $\unrhd$ is a d-averaging rule, with $u=v$. Futhermore, the mapping $u$ is unique up to a positive linear transformation.

Proof. It is easy to check that all conditions are necessary. We only prove the sufficiency. As in Theorem $1, \succsim$ is a scoring rule. Consider now two departments $\left(f_{1}, \ldots, f_{k}\right)$ and $\left(g_{1}, \ldots, g_{\ell}\right)$. Define $f=\sum_{i=1}^{k} f_{i}$ and $g=\sum_{i=1}^{\ell} g_{i}$. By Homogeneity and Totality,

$$
\left(f_{1}, \ldots, f_{k}\right) \triangleq(\underbrace{f_{1}, \ldots, f_{1}}_{\ell}, \ldots, \underbrace{f_{k}, \ldots, f_{k}}_{\ell}) \triangleq(\ell \cdot f, \underbrace{\mathbf{0}, \ldots, \mathbf{0}}_{\ell k-1})
$$


and

$$
\left(g_{1}, \ldots, g_{\ell}\right) \triangleq(\underbrace{g_{1}, \ldots, g_{1}}_{k}, \ldots, \underbrace{g_{\ell}, \ldots, g_{\ell}}_{k}) \triangleq(k \cdot g, \underbrace{\mathbf{0}, \ldots, \mathbf{0}}_{\ell k-1}) .
$$

So, by Consistency, $\left(f_{1}, \ldots, f_{k}\right) \unrhd\left(g_{1}, \ldots, g_{\ell}\right)$ iff $\ell \cdot f \succsim k \cdot g$ iff $\ell \sum_{i=1}^{k} \sum_{x \in \mathbb{N}} f_{i}(x) u(x) \geq$ $k \sum_{i=1}^{\ell} \sum_{x \in \mathbb{N}} g_{i}(x) u(x)$ iff

$$
\frac{1}{k} \sum_{i=1}^{k} \sum_{x \in \mathbb{N}} f_{i}(x) u(x) \geq \frac{1}{\ell} \sum_{i=1}^{\ell} \sum_{x \in \mathbb{N}} g_{i}(x) u(x) .
$$

The only difference between Theorems 1 and 3 is that Homogeneity is substituted for Dummy Scientist. In our opinion, none of these two conditions is more appealing or reasonable than the other. There can be circumstances where we want to know which department has the larger impact and we then impose Dummy Scientist. There are other circumstances where we want to determine the department in which scientists are most productive, on average; we then impose Homogeneity.

Scoring rules and averaging rules depend on a single parameter: the mapping $u$ that can take any shape. Of course, in most circumstances, we will need a nondecreasing mapping. This can be achieved by imposing some kind of monotonicity condition (for instance condition CDNH as in Marchant [2009b]). In some circumstances, we might wish to work with a non-constant mapping $u$ or a linear one, etc. It is of course possible to impose some additional conditions on top of those of Theorem 1 or 3 in order to restrict the shape of the mapping $u$. Such conditions have been discussed in Marchant [2009b].

\section{$5 \quad$ Independence of the axioms}

We now prove that the axioms used in Theorems 1 and 3 are logically independent, that is, if we remove any axiom from the statement of one theorem, then that theorem no longer holds. This will establish the parsimony of our results.

We start with the independence of the axioms in Theorem 1. To this end, we provide for each axiom a counter-example (a pair of rankings) satisfying all axioms but that specific axiom.

Consistency. Define $\succsim$ as a s-scoring rule with $u(x)=1$ for all $x \in \mathbb{N}$ and $\unrhd$ as a d-scoring rule with $v(x)=x$ for all $x \in \mathbb{N}$. Consider then two departments each consisting of one scientist with a single publication but with different number of citations. The two scientists are equivalent but the departments are not, thereby violating Consistency. 
Totality. Define $\succsim$ as a s-scoring rule with any $u$ and rank the departments in decreasing order of $\sum_{i=1}^{k}\left[\sum_{x \in \mathbb{N}} f_{i}(x) u(x)\right]^{2}$.

Dummy. Define $\succsim$ as a s-scoring rule and $\unrhd$ as a d-averaging rule. Adding a dummy scientist to a department worsens its position in the ranking.

Archimedeanness. Define $\succsim$ by $f \succsim g$ iff

- either $\sum_{x \in \mathbb{N}^{+}} f(x)>\sum_{x \in \mathbb{N}^{+}} g(x)$

- or $\sum_{x \in \mathbb{N}^{+}} f(x)=\sum_{x \in \mathbb{N}^{+}} g(x)$ and $f(0) \geq g(0)$.

Define $\unrhd$ by $\left(f_{1}, \ldots, f_{k}\right) \unrhd\left(g_{1}, \ldots, g_{\ell}\right)$ iff

- either $\sum_{i=1}^{k} \sum_{x \in \mathbb{N}^{+}} f_{i}(x)>\sum_{i=1}^{\ell} \sum_{x \in \mathbb{N}^{+}} g_{i}(x)$

- or $\sum_{i=1}^{k} \sum_{x \in \mathbb{N}^{+}} f_{i}(x)=\sum_{i=1}^{\ell} \sum_{x \in \mathbb{N}^{+}} g_{i}(x)$ and $f(0) \geq g(0)$.

To see that $\succsim$ does not satisfy Archimedeanness, define $e=\mathbf{1}_{1}, h=\mathbf{1}_{2}$, $f=2 \cdot \mathbf{1}_{0}$ and $g=\mathbf{1}_{0}$. We have $f \succ g$ and $e \prec h$. There is no $n$ such that $e+n \cdot f \succsim h+n \cdot g$.

We now turn to the independence of the axioms in Theorem 3. They are minor variations of the examples used above.

Consistency. Define $\succsim$ as a s-scoring rule with $u(x)=1$ for all $x \in \mathbb{N}$ and $\unrhd$ as a d-averaging rule with $v(x)=x$ for all $x \in \mathbb{N}$. Consider then two departments each consisting of one scientist with a single publication but with different number of citations. The two scientists are equivalent but the departments are not, thereby violating Consistency.

Totality. Define $\succsim$ as a s-scoring rule with any $u$ and rank the departments in decreasing order of $\frac{1}{k} \sum_{i=1}^{k}\left[\sum_{x \in \mathbb{N}} f_{i}(x) u(x)\right]^{2}$.

Homogeneity. Define $\succsim$ as a s-scoring rule and $\unrhd$ as a d-scoring rule. Doubling the size of a department by cloning all its members will result in an improvement of its position in the ranking whilst Homogeneity says it should not matter.

Archimedeanness. Define $\succsim$ by $f \succsim g$ iff

- either $\sum_{x \in \mathbb{N}^{+}} f(x)>\sum_{x \in \mathbb{N}^{+}} g(x)$

- or $\sum_{x \in \mathbb{N}^{+}} f(x)=\sum_{x \in \mathbb{N}^{+}} g(x)$ and $f(0) \geq g(0)$.

Define $\unrhd$ by $\left(f_{1}, \ldots, f_{k}\right) \unrhd\left(g_{1}, \ldots, g_{\ell}\right)$ iff 
- either $\frac{1}{k} \sum_{i=1}^{k} \sum_{x \in \mathbb{N}^{+}} f_{i}(x)>\frac{1}{k} \sum_{i=1}^{\ell} \sum_{x \in \mathbb{N}^{+}} g_{i}(x)$

- or $\frac{1}{k} \sum_{i=1}^{k} \sum_{x \in \mathbb{N}^{+}} f_{i}(x)=\frac{1}{k} \sum_{i=1}^{\ell} \sum_{x \in \mathbb{N}^{+}} g_{i}(x)$ and $f(0) \geq g(0)$.

To see that $\succsim$ does not satisfy Archimedeanness, define $e=\mathbf{1}_{1}, h=\mathbf{1}_{2}$, $f=2 \cdot \mathbf{1}_{0}$ and $g=\mathbf{1}_{0}$. We have $f \succ g$ and $e \prec h$. There is no $n$ such that $e+n \cdot f \succsim h+n \cdot g$.

\section{Some comments on Totality}

Totality has two different facets. On the one hand, it imposes that the identity of the scientists does not matter. In other words, we can permute the order of the scientists in the vector representing a department without affecting the position of the department in the ranking. For instance, $\left(3 \cdot \mathbf{1}_{3}, \mathbf{0}, 6 \cdot \mathbf{1}_{10}\right) \triangleq\left(6 \cdot \mathbf{1}_{10}, 3 \cdot \mathbf{1}_{3}, \mathbf{0}\right)$. On the other hand, Totality is related to the distribution of papers among scientists: it imposes that this distribution plays no role. For instance, $\left(3 \cdot \mathbf{1}_{3}, \mathbf{0}, 6 \cdot \mathbf{1}_{10}\right) \triangleq$ $\left(\mathbf{1}_{3}+2 \cdot \mathbf{1}_{10}, \mathbf{1}_{3}+2 \cdot \mathbf{1}_{10}, \mathbf{1}_{3}+2 \cdot \mathbf{1}_{10}\right)$. It therefore seems desirable to split Totality into two more elementary conditions named Anonymity and Paper Transfer: the former about the identity of the scientists and the latter about the distribution among the scientists.

A 8 Anonymity. For every permutation $\sigma$ of $\{1, \ldots, k\}$, we have $\left(f_{1}, \ldots, f_{k}\right) \triangleq$ $\left(f_{\sigma(1)}, \ldots, f_{\sigma(k)}\right)$.

In other words, if we permute the order of the scientists in the department, we obtain a new department indifferent to the original one. This condition is very appealing but one might want to reject it if, for instance, the head of the department is always in first position in the vector and if one wants to give him more weight.

A 9 Paper Transfer. For every $x \in \mathbb{N}$, we have $\left(f_{1}+\mathbf{1}_{x}, f_{2}, \ldots, f_{k}\right) \triangleq\left(f_{1}, f_{2}+\right.$ $\left.\mathbf{1}_{x}, \ldots, f_{k}\right)$.

Put differently, we may transfer papers between the first two scientists in the department without impacting the position of the department. As a consequence, a department consisting of two scientists where the first one has 10 papers, each one cited 10 times and the second one has no paper is equivalent to a department of the same size where both have 5 papers, each one cited 10 times. In some circumstances, it is of course possible to argue that the more uniform department is better. And in other circumstances, one might also say that the less uniform department is better because one excellent scientist has more impact than two mediocre ones. Notice that the condition Paper Transfer is different from the condition called Transfer or Transferability in [Marchant, 2009b, Bouyssou and 
Marchant, 2010], which is about the transfer of citations between papers of a single scientist.

We now show that the conjunction of Anonymity and Paper Transfer is equivalent to Totality.

Proposition 2 The relation $\unrhd$ satisfies Anonymity and Paper Transfer if and only if $\unrhd$ satisfies Totality.

Proof. We prove only $\Rightarrow$. Let $f$ and $g$ be such that $\sum_{i=1}^{k} f_{i}=\sum_{i=1}^{k} g_{i}$. We must prove that $\left(f_{1}, \ldots, f_{k}\right) \triangleq\left(g_{1}, \ldots, g_{k}\right)$. If we apply Paper Transfer $\left(\sum_{x \in \mathbb{N}} f_{2}(x)\right)$ times, we find $\left(f_{1}, f_{2}, f_{3}, \ldots, f_{k}\right) \triangleq\left(f_{1}+f_{2}, \mathbf{0}, f_{3}, \ldots, f_{k}\right)$. We can then permute scientists 2 and 3 and we obtain $\left(f_{1}, f_{2}, f_{3}, \ldots, f_{k}\right) \triangleq\left(f_{1}+f_{2}, \mathbf{0}, f_{3}, \ldots, f_{k}\right) \triangleq$ $\left(f_{1}+f_{2}, f_{3}, \mathbf{0}, \ldots, f_{k}\right)$. We apply again Paper Transfer $\left(\sum_{x \in \mathbb{N}} f_{3}(x)\right)$ times and we find $\left(f_{1}, f_{2}, f_{3}, \ldots, f_{k}\right) \triangleq\left(f_{1}+f_{2}, f_{3}, \mathbf{0}, \ldots, f_{k}\right) \triangleq\left(f_{1}+f_{2}+f_{3}, \mathbf{0}, \mathbf{0}, \ldots, f_{k}\right)$. It is clear that if we iterate this process, we will obtain $\left(f_{1}, f_{2}, f_{3}, \ldots, f_{k}\right) \triangleq$ $\left(\sum_{i=1}^{k} f_{i}, \mathbf{0}, \mathbf{0}, \ldots, \mathbf{0}\right)$. Similarly, we obtain $\left(g_{1}, g_{2}, g_{3}, \ldots, g_{k}\right) \triangleq\left(\sum_{i=1}^{k} g_{i}, \mathbf{0}, \mathbf{0}, \ldots, \mathbf{0}\right)$. Since $\sum_{i=1}^{k} f_{i}=\sum_{i=1}^{k} g_{i}$, we finally obtain $\left(f_{1}, \ldots, f_{k}\right) \triangleq\left(g_{1}, \ldots, g_{k}\right)$.

Thanks to this proposition, Totality can be replaced by Anonymity and Paper Transfer in the statements of Theorems 1 and 3 .

It is worth noticing that the first and the second scientists of every department play a special role in the statement of Paper Transfer. The condition is indeed about paper transfers from the first one to the second one or the converse; the other scientists are not involved. This awkward asymmetry can be avoided by imposing a slightly different condition, as follows.

A 10 Paper Transfer*. For every $x \in \mathbb{N}$ and every $i, j \in\{1, \ldots, k\}$, we have $\left(f_{1}, \ldots, f_{i}+\mathbf{1}_{x}, \ldots, f_{j}, \ldots, f_{k}\right) \triangleq\left(f_{1}, \ldots, f_{i}, \ldots, f_{j}+\mathbf{1}_{x}, \ldots, f_{k}\right)$.

With this condition, the transfer takes place between any pair of scientists in the department. Unfortunately, it is easy to see that this condition implies Anonymity and is therefore equivalent to Totality We therefore cannot use it for splitting (or factorizing) Totality into more elementary (single-faceted) conditions. We did not succeed in finding a totaly satisfactory factorization of Totality. The best one we found is presented in Proposition 2.

\section{Discussion}

Our first result is about the relation between Independence and Consistency. Consistency is a very compelling condition. Any pair of rankings violating Consistency 
would be very suspicious. Independence is quite compelling as well but not to the same extent. We show that, under Totality, Consistency implies Independence, thereby reinforcing the attractiveness of Independence. The question is then: why should we abide by Totality? We have no clear answer to this question, but almost all papers about rankings of departments accept it. In our opinion, rejecting Totality is a very non-standard (although not unreasonable) attitude and it should be motivated by strong arguments.

Nevertheless, even without knowing the answer to the question whether Totality should hold, our analysis (and that of Waltman and van Eck [2009]) has clear implications for the practice: using the h-index for ranking scientists and departments under Totality (as in Kinney [2007]) is very weird, because it violates Consistency and Independence.

If we adopt Totality and Consistency, we have shown that, under few extra plausible conditions, the only possible rankings of scientists are scoring rules and the only possible rankings of departments are scoring rules or averaging rules. The distinction between averaging and scoring rules for departments results from the adoption of Homogeneity or Dummy Scientist. The former emphasizes total production, the latter, average production (or productivity).

In a next stage, we plan to further analyze the implications of Consistency when simultaneously ranking scientists and departments, in order to overcome some of the limitations of the present paper.

- Endogeneous treatment of coauthorship. When we consider the publications of a department, it is very likely that many of them will be coauthored by several scientists affiliated to that very same department. This is of course something that should be taken into account if we want to rank order departments on the basis of the publication/citation records of its members. In this paper, we have decided to ignore this problem. An easy solution would be to consider that the problem has been addressed beforehand and that it has been decided to share publications with several authors among them according to some scheme. For instance, a paper signed by $n$ scientists counts for $1 / n$ in the publication/citation records of each coauthor as in [Coupé, 2003]. Many other sharing schemes are possible and can perhaps better reflect the traditions within some discipline. It is for instance possible to give more weight to the first author ${ }^{7}$.

A more interesting approach would be to handle this problem endogeneously, deriving a sharing scheme or another way of coping with coauthorship not from a prior decision but from our set of axioms. To this end, we could for

\footnotetext{
${ }^{7}$ This should not be applied to the present paper since we have explicitely stated that authors are listed alphabetically.
} 
instance use a condition imposing that a group of scientists cannot benefit from systematically and artificially putting each other's name on all their publications. Notice that this would require a richer data structure than the simple citation distribution that we used in this paper. We would probably need a graph structure similar to the one in Bouyssou and Marchant [2010].

- Endogeneous treatment of multiple affiliations. We did not touch upon this problem yet but it is clearly a difficulty that anyone will encounter when ranking departments. It can of course be treated exogeneously just like the problem of coauthorship. For instance, we can a priori decide that a department gets only $1 / N$ of a paper when the author has $N$ affiliations. We then need to "correct" the data (the citation distribution) before constructing the ranking. A better approach would be to derive a solution for this problem from our set of axioms. Here again, a graph structure similar to the one in Bouyssou and Marchant [2010] would probably be necessary.

We mention a last research direction: what are the consequences of Consistency without Totality? Although most researchers implicitly accept Totality, we think this might be an interesting question.

\section{Acknowledgements}

We are indebted to Ludo Waltman for his comments about a previous version. The work of Denis Bouyssou was partially supported by a grant from the Agence Nationale de la Recherche (Project ComSoc, ANR-09-BLAN-0305). This support is gratefully acknowledged.

\section{References}

T. R. Anderson, R. K. S. Hankin, and P. D. Killworth. Beyond the Durfee square: Enhancing the h-index to score total publication output. Scientometrics, 76(3): $577-588,2008$.

R. Arencibia-Jorge, I. Barrios-Almaguer, S. Fernández-Hernández, and R. Carvajal-Espino. Applying successive h-indices in the institutional evaluation: A case study. Journal of the American Society for Information Science and Technology, 59:155-157, 2008.

D. Bouyssou and T. Marchant. Consistent bibliometric rankings of authors and of journals. Journal of Informetrics, 4:365-378, 2010. 
D. Bouyssou and T. Marchant. Bibliometric rankings of journals based on impact factors: An axiomatic approach. Journal of Informetrics, 5(1):75-86, 2011.

T. Coupé. Revealed performances: Worldwide rankings of economists and economics departments, 1990-2000. Journal of the European Economic Association, (1):1309-1345, 2003.

R. Dusansky and C. J. Vernon. Rankings of U.S. economics departments. Journal of Economic Perspectives, 12:157-170, 1998.

L. Egghe. Theory and practise of the $g$-index. Scientometrics, 69:131-152, 2006a.

L. Egghe. An improvement of the h-index: The g-index. ISSI Newsletter, 2(1): 8-9, 2006b.

J. E. Hirsch. An index to quantify an individual's scientific research output. Proceedings of the National Academy of Sciences, 102:16569-16572, 2005.

B. Jin, L. Liang, R. Rousseau, and L. Egghe. The R- and AR-indices: Complementing the h-index. Chinese Science Bulletin, 52(6):855—863, 2007.

A. L. Kinney. National scientific facilities and their science impact on nonbiomedical research. Proceedings of the National Academy of Sciences, 104:1794317947, 2007.

H. W. Kinnucan and G. Traxler. Ranking agricultural economics departments by AJAE page counts: A reappraisal. Agricultural and Resource Economics Review, 23:194-199, 1994.

M. Kosmulski. A new Hirsch-type index saves time and works equally well as the original h-index. ISSI Newsletter, 2(3):4-6, 2006.

R. D. Luce. Utility of gains and losses: measurement-theoretical and experimental approaches. Lawrence Erlbaum Associates, Mahwah, New Jersey, 2000.

T. Marchant. An axiomatic characterization of the ranking based on the $h$-index and some other bibliometric rankings of authors. Scientometrics, 80:325-342, 2009a.

T. Marchant. Score-based bibliometric rankings of authors. Journal of the American Society for Information Science and Technology, 60:1132-1137, 2009b.

A. Schubert. Successive h-indices. Scientometrics, 70:201-205, 2007.

N. J. van Eck and L. Waltman. Generalizing the h- and g-indices. Journal of Informetrics, 2(4):263-271, 2008. 
A. F. J. van Raan. Comparison of the Hirsch-index with standard bibliometric indicators and with peer judgment for 147 chemistry research groups. Scientometrics, 67:491-502, 2006.

L. Waltman and N. J. van Eck. A taxonomy of bibliometric performance indicators based on the property of consistency. Technical report, ERIM, 2009. http://publishing.eur.nl/ir/repub/asset/15182/ERS-2009-014-LIS.pdf.

L. Waltman, N. J. van Eck, T. N. van Leeuwen, M. S. Visser, and A. F. J. van Raan. Towards a new crown indicator: Some theoretical considerations. Journal of Informetrics, 5:37-47, 2011. 